LAWRENCE LIVERMORE N A T IO N A L LABORATORY

\section{Optimization of Aperiodic Waveguide Mode Converters}

G. J. Burke, D. A. White, C. A. Thompson

December 14, 2004

2005 IEEE/ACES International Conference on Wireless Communications and Applied Computational Electromagnetics Honolulu, $\mathrm{HI}$, United States

April 3, 2004 through April 7, 2004 
This document was prepared as an account of work sponsored by an agency of the United States Government. Neither the United States Government nor the University of California nor any of their employees, makes any warranty, express or implied, or assumes any legal liability or responsibility for the accuracy, completeness, or usefulness of any information, apparatus, product, or process disclosed, or represents that its use would not infringe privately owned rights. Reference herein to any specific commercial product, process, or service by trade name, trademark, manufacturer, or otherwise, does not necessarily constitute or imply its endorsement, recommendation, or favoring by the United States Government or the University of California. The views and opinions of authors expressed herein do not necessarily state or reflect those of the United States Government or the University of California, and shall not be used for advertising or product endorsement purposes. 


\title{
Optimization of Aperiodic Waveguide Mode Converters *
}

\author{
G. J. Burke, D. A. White, C. A. Thompson \\ Lawrence Livermore National Laboratory, Livermore, CA 94550 \\ email: burke2@llnl.gov, white37@llnl.gov, thompson53@llnl.gov
}

\section{Introduction}

Previous studies by Haq, Webb and others have demonstrated the design of aperiodic waveguide structures to act as filters and mode converters [1,2]. These aperiodic structures have been shown to yield high efficiency mode conversion or filtering in lengths considerably shorter than structures using gradual transitions and periodic perturbations. The design method developed by Haq and others has used mode-matching models [3] for the irregular, stepped waveguides coupled with computer optimization to achieve the design goal using a Matlab optimization routine.

Similar designs are described here, using a mode matching code written in Fortran and with optimization accomplished with the downhill simplex method with simulated annealing using an algorithm from the book Numerical Recipes in Fortran [4]. Where Haq et al. looked mainly for waveguide shapes with relatively wide cavities, we have sought lower profile designs. It is found that lower profiles can meet the design goals and result in a structure with lower Q. In any case, there appear to be very many possible configurations for a given mode conversion goal, to the point that it is unlikely to find the same design twice. Tolerance analysis was carried out for the designs to show edge sensitivity and Monte Carlo degradation rate. The mode matching code and mode conversion designs were validated by comparison with FDTD solutions for the discontinuous waveguides.

\section{Optimization for design of waveguide structures}

In the mode matching solution the fields in two waveguides forming a junction are expanded in a sum of modes and the continuity of $\mathrm{E}$ and $\mathrm{H}$ fields is enforced as integrals weighted with the mode functions. The procedure described in [3] yields the scattering matrix for a junction. The scattering matrix for a sequence of junctions, taking account of all interactions, can then be obtained by cascading individual scattering matrices [5]. We found that rough results could be obtained with modes $\mathrm{TE}_{10}$ and $\mathrm{TE}_{30}$ in the input guide, where only $\mathrm{TE}_{10}$ was above cutoff, and the number of modes in other sections scaled in proportion to width. Higher accuracy was obtained with modes up to $\mathrm{TE}_{90}$ in the input and proportional scaling. The mode matching solution took about 0.03 to 0.1 seconds for a single frequency with 20 waveguide junctions on a $1.7 \mathrm{GHz}$ Pentium computer using modes up to $\mathrm{TE}_{90}$ in the input. These wave guide structures were also modeled with a 2D FDTD code with a plane of symmetry. The FDTD solution could take 20 minutes to several hours to yield all frequencies for a given waveguide configuration, but could offer options for more generality in the model if needed. Both mode matching and FDTD codes were coupled with optimization, but the mode matching is of course much nicer to use.

To synthesize waveguide mode converters the solver was coupled with an optimization

* This work was performed under the auspices of the U. S. Department of Energy by the University of California, Lawrence Livermore National Laboratory under Contract No. W-7405-Eng-48. 
code that used the downhill simplex method with simulated annealing [4]. For an $N$ parameter space, a simplex is a figure of $N+1$ vertices and connecting edges, in this case $N+1$ different configurations of the waveguide sections. The objective function is evaluated at each vertex. Then the algorithm may reflect the highest (worst) vertex relative to the others, and may also expand or contract the simplex. The initial simplex was generated by entering an initial waveguide profile and generating $N$ additional profiles with random perturbations. Simulated annealing adds a randomness to simulate a metal cooling to its lowest energy state. It is stated in [4] that an annealing schedule must be chosen for each particular problem. We usually just optimized with zero temperature from the initial random simplex, and if the result looked promising, then raised the temperature and lowered it to see if a better solution could be found.

Two mode converter designs are shown in Fig. 1 through 4 for a goal to optimize $\mathrm{TE}_{10}$ output at $9.6,9.7$ and $9.8 \mathrm{GHz}$ and $\mathrm{TE}_{30}$ output at $10 \mathrm{GHz}$ for $\mathrm{TE}_{10}$ input. The cost function was the product of the output powers for the designated modes and frequencies. The waveguide had 20 sections of $1 \mathrm{~cm}$ length that were only adjusted in width. Both designs meet the goals fairly well, although the second has a lower tolerance. The result of a FDTD solution for the final design is included in Fig. 1, and is in good agreement with the mode matching result. A design to pass $\mathrm{TE}_{10}$ at 9.7 and $10.3 \mathrm{GHz}$ and convert to $\mathrm{TE}_{30}$ at $10 \mathrm{GHz}$ is shown in Fig. 5 and 6 . In this case the waveguide had 10 sections that were adjusted in both width and length. For this goal we were unable to find a good low profile design with 20 sections adjusted in width. We did find a good solution with $21 \mathrm{~cm}$ maximum width, but it had a very low tolerance. A good low profile design was also found with 40 sections of 0.5 $\mathrm{cm}$ length adjusted in width.

\section{Conclusion}

The mode matching solution for discontinuous waveguides, coupled with the downhill simplex method of optimization and simulated annealing provides an efficient means of designing waveguide structures as mode converters and filters. In many cases good results were obtained in very reasonable computer time by using a minimal number of modes. When a promising result was obtained the number of modes could be increased to ensure accuracy. A similar strategy was described in [2]. There may be many possible solutions for a given goal and many not so good relative minima, but the method of Simulated Annealing was helpful in finding a good design.

\section{References}

[1] T. ul Haq, K. J. Webb and N. C. Gallagher, "Scattering Optimization Method for the Design of Compact Mode Converters for Waveguides," IEEE Trans. Microwave Theory Tech., vol. 43, no. 3, pp. 559-565, March 1995.

[2] M. C. Yang, J. H. Li and K. J. Webb, "Functional Waveguide Mode Transformers," IEEE Trans. Microwave Theory Tech., vol. 52, no. 1, pp. 161-169, Jan. 2004.

[3] A. Wexler, "Solution of Waveguide Discontinuities by Modal Analysis," IEEE Trans. Microwave Theory Tech., vol. 15, no. 9, pp. 508-516, Sept. 1967.

[4] W. H. Press, S. A. Teukolsky, W. T. Vetterling and B P. Flannery, Numerical Recipes in Fortran (alsoNumerical Recipes in C), Cambridge Univ. Press, New York, 1994.

[5] T. S. Chu and T. Itoh, "Generalized Scattering Matrix Method for Analysis of Cascaded and Offset Microstrip Step Discontinuities", IEEE Trans. Microwave Theory Tech., vol. 34, no. 2, pp. 280-284, Feb. 1986. 

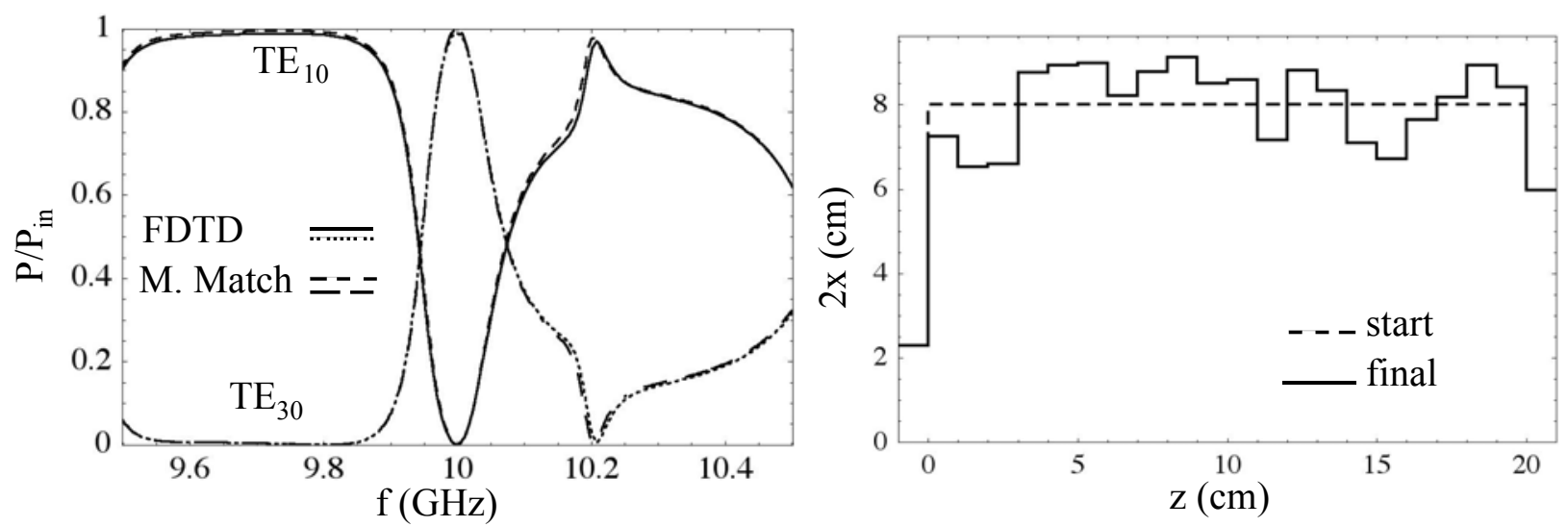

Fig. 1. Waveguide mode converter optimized for $\mathrm{TE}_{10}$ output at $9.6,9.7$ and $9.8 \mathrm{GHz}$ and for $\mathrm{TE}_{30}$ output at $10 \mathrm{GHz}$ for $\mathrm{TE}_{10}$ in the input guide. The optimization was started with a flat initial profile with width of $8 \mathrm{~cm}$ and 20 adjustable sections with $1 \mathrm{~cm}$ lengths. The " $2 x$ " scale shows the full width of the waveguide.
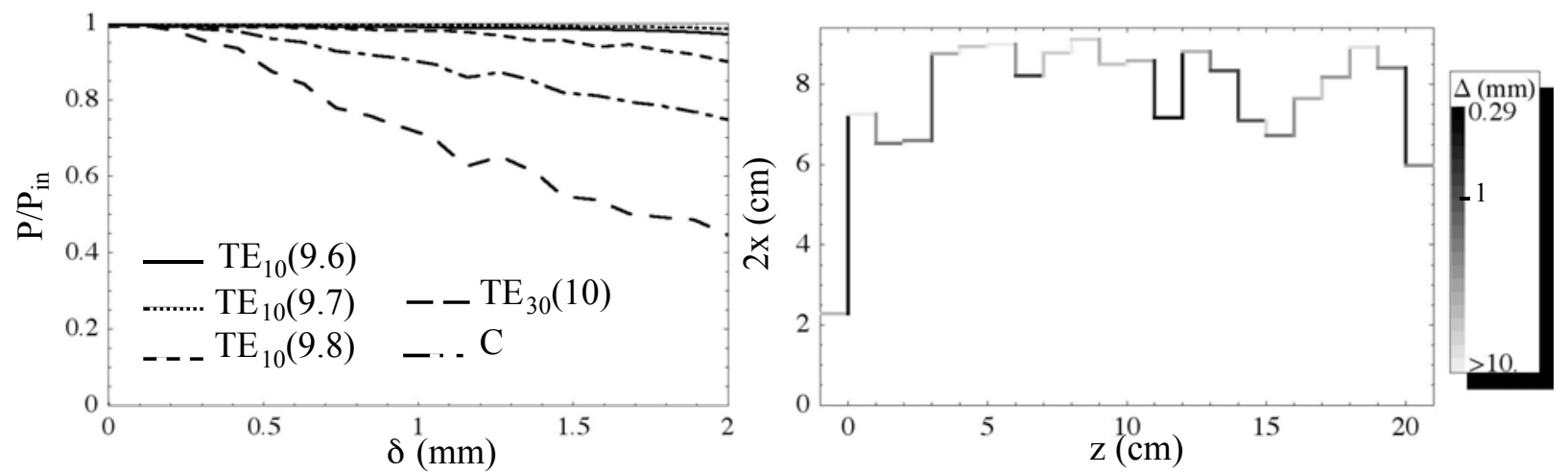

Fig. 2. Dimension tolerance plots for the waveguide in Fig. 1, including Monte Carlo analysis for error range $\pm \delta$ and edge sensitivity $\Delta$ for one percent degradation in the cost function $C$ where $C=$ $\left[P_{\mathrm{TE} 10}(9.6) P_{\mathrm{TE} 10}(9.7) P_{\mathrm{TE} 10}(9.8) P_{\mathrm{TE} 30}(10)\right]^{1 / 4}$ and $P_{\mathrm{TEn} 0}(f)$ indicates the normalized power in mode $\mathrm{TE}_{n 0}$ at $\mathrm{f} \mathrm{GHz}$.
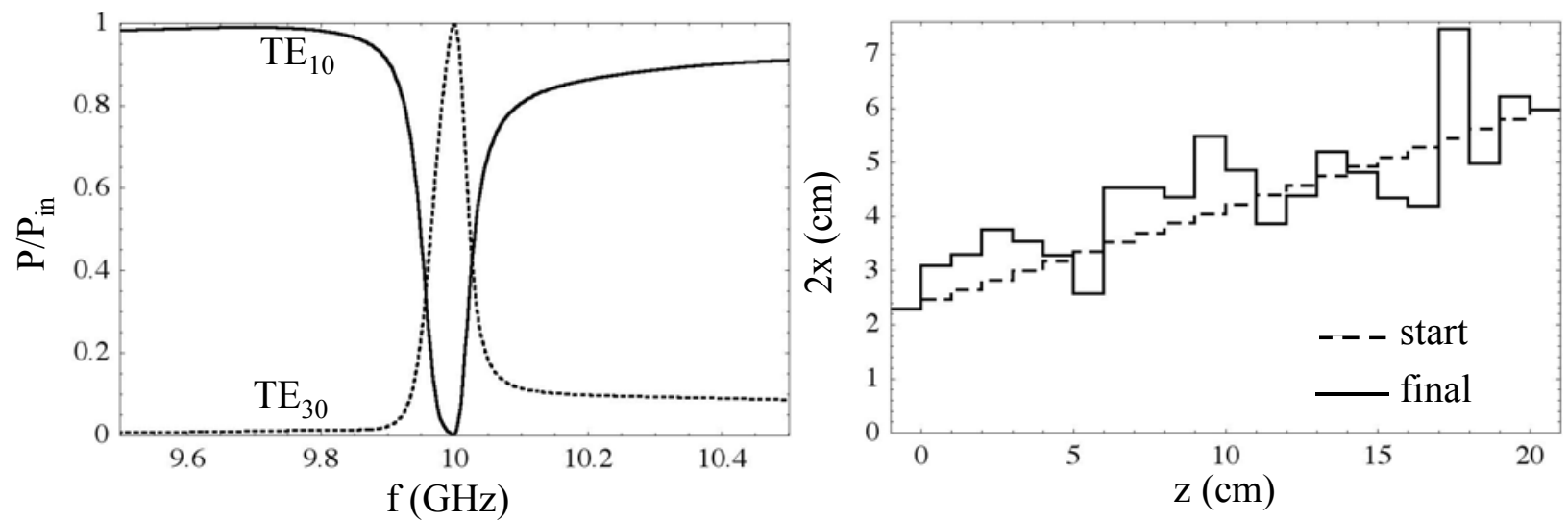

Fig. 3. Waveguide mode converter optimized for $\mathrm{TE}_{10}$ output at $9.6,9.7$ and $9.8 \mathrm{GHz}$ and for $\mathrm{TE}_{30}$ output at $10 \mathrm{GHz}$ for $\mathrm{TE}_{10}$ in the input guide. The optimization was started with a tapered initial profile. 

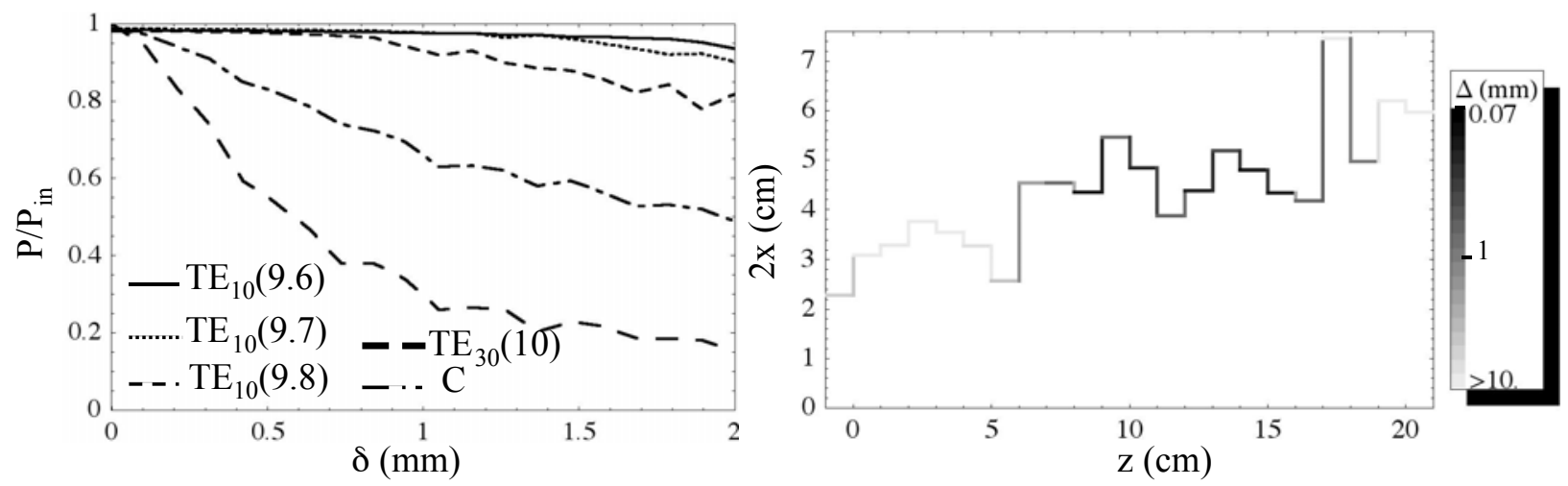

Fig. 4. Dimension tolerance plots for the waveguide in Fig. 3, including Monte Carlo analysis for error range $\pm \delta$ and edge sensitivity $\Delta$ for one percent degradation in the cost function $C$.
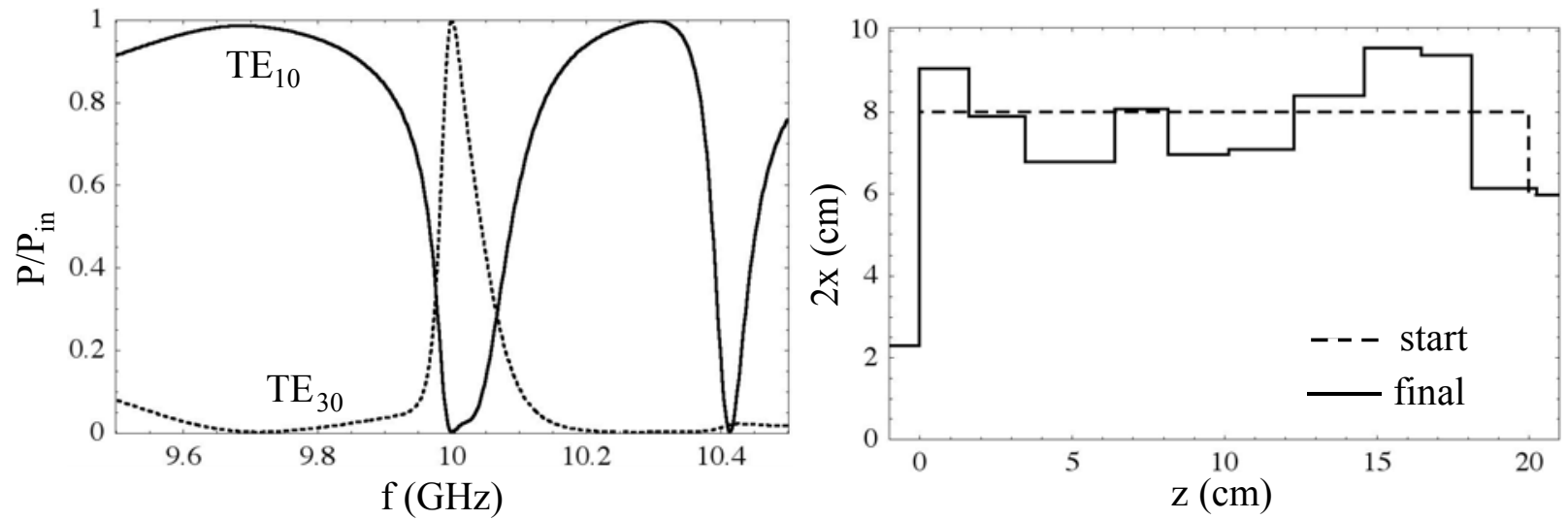

Fig. 5. Waveguide mode converter optimized for $\mathrm{TE}_{10}$ output at 9.7 and $10.3 \mathrm{GHz}$ and $\mathrm{TE}_{30}$ output at 10 $\mathrm{GHz}$ for $\mathrm{TE}_{10}$ in the input guide. The optimization was started with a flat initial profile with width of $8 \mathrm{~cm}$, and 10 sections with both width and length varied.
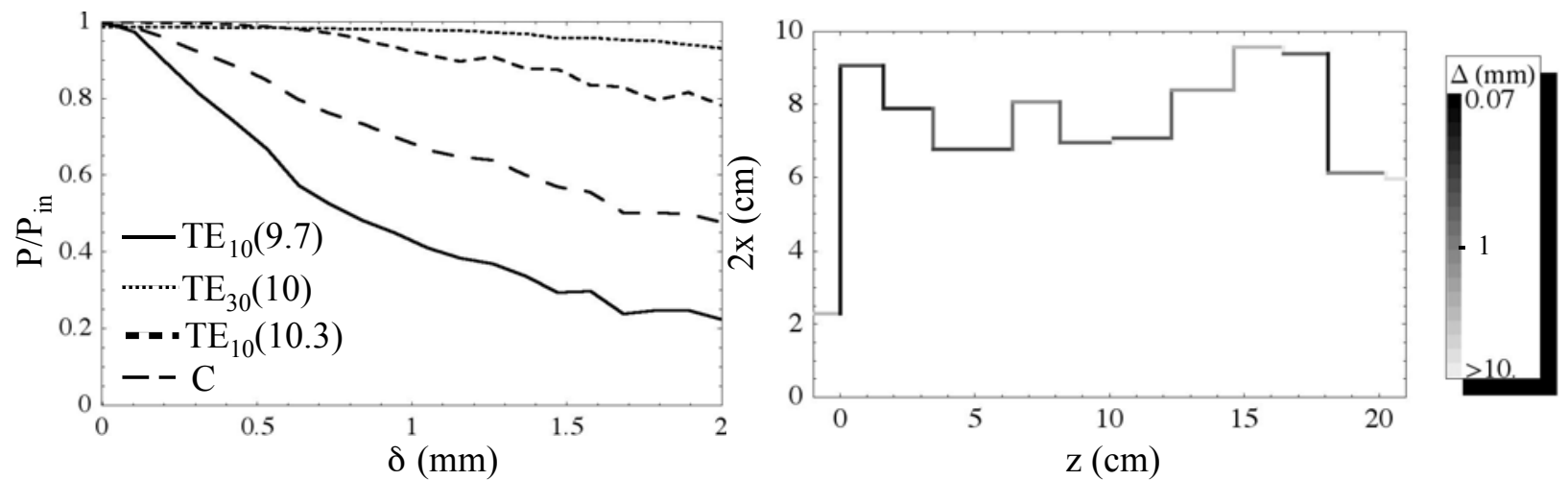

Fig. 6 . Dimension tolerance plots for the waveguide in Fig. 5, including Monte Carlo analysis for error range $\pm \delta$ and edge sensitivity $\Delta$ for one percent degradation in the cost function $C$. 